\title{
Review: hand symptom diagrams, weak thumb abduction, and hypalgesia are helpful in diagnosing carpal tunnel syndrome
}

D'Arcy CA, McGee S. Does this patient have carpal tunnel syndrome? JAMA 2000 Jun 21;283:3110-7.

\author{
QUESTION: What is the accuracy of the history and physical examination in diagnosing \\ carpal tunnel syndrome (CTS)?
}

\section{Data sources}

Studies were identified by searching Medline (1966 to February 2000) with the terms carpal tunnel syndrome, diagnosis, Tinel, Hoffman, and Phalen. References of relevant articles were reviewed. The search was limited to English language studies.

\section{Study selection}

Studies were selected if they addressed the diagnosis of CTS in adults; patients presented with symptoms suggestive of CTS; a clear description of the physical examination was provided; the results of the physical examination and electrodiagnostic studies were apparently assessed independently; and numbers needed to calculate sensitivity, specificity, and likelihood ratios (LRs) were available.

\section{Data extraction}

2 independent reviewers extracted data on history, physical findings, and CTS diagnosis; sensitivity, specificity, and positive and negative likelihood ratios were calculated.

\section{Main results}

12 articles met the selection criteria. For summary LR measures, data were combined using the Dersimonian and Laird random effects model. Of the 17 history and physical examination manoeuvres studied, the table shows the tests for which $\geqslant 2$ studies were reported and for which the 95\% CIs excluded 1.0 for the + LR or -LR. The classic or probable Katz hand diagram (1 study) is also shown because of its strong clinical interest.

\section{Conclusions}

Among 17 findings on the history and physical examination, hypalgesia to pin prick, increased anteroposterior to mediolateral wrist dimension (square wrist), and a classic or probable hand diagram are most discriminating for ruling in carpal tunnel syndrome (CTS). The normal square wrist test, hand symptom diagram, and thumb abduction strength test are the most useful indicators for ruling out CTS.

\section{COMMENTARY}

What are the clinical implications of the findings in the review from D'Arcy and McGee? Let us consider a patient whom we judge to have a pretest probability of $50 \%$ for CTS. Applying the hand symptom diagram in such a patient shifts this probability, if the symptom diagram is positive, to a post-test probability of $71 \%$; if the diagram is negative, the probability shifts to $33 \%$. If the purpose is to decide whether to do electrodiagnostic testing, use of the diagram may not have influenced our decision. However, if we sequentially do thumb abduction strength testing, the probabilities change to $82 \%$ and $20 \%$, respectively. These values are now close to the thresholds for deciding whether to do further investigations or to defer them. Confirmatory findings from some of the other clinical tests noted above would provide additional clarification.

The magnitude of the LRs for the individual tests reported here by the authors are generally not sufficient to change pretest probability to a clinically important degree. The authors point out that little research has been done on the usefulness of tests in combination. However, considering their properties in this way shows that these tests are likely to be more helpful in decision making when used in sequence.

Few studies on clinical diagnosis in mild or early CTS exist; thus, the current findings are not directly applicable to the spectrum of CTS encountered in primary care.

Although the literature search for the review by D'Arcy and McGee could have been more comprehensive and the authors could have been more explicit about their reviewing methods, ${ }^{2}$ they still provide a clear basis for considering the merits and limitations of our approaches to clinical diagnosis of CTS. Ted Haines, MD, MSc, DOHS McMaster University Hamilton, Ontario, Canada Carol Kennedy, PT University of Toronto, Toronto, Ontario, Canada

1. Sackett DL, Straus SE, Richardson WS, et al. Evidence-based medicine. How to practice and teach EBM. 2nd edition. Edinburgh: Churchill Livingstone; 2000.

2. Oxman AD, Cook DJ, Guyatt GH. Users' guides to the medical literature. VI. How to use an overview. Evidence-based medicine working group. JAMA 1994;272:1367-71.

USA.Fax + 1206685

9397.

Test characteristics for history and physical examination for diagnosing carpal tunnel syndrome*

\begin{tabular}{|c|c|c|c|c|c|}
\hline Test & Number of studies & Sensitivity range & Specificity range & $+\operatorname{LR}(95 \% \mathrm{Cl})$ & -LR (Cl) \\
\hline Square wrist sign & 2 & $47 \%$ to $69 \%$ & $73 \%$ to $83 \%$ & 2.7 (2.2 to 3.4$)$ & $0.5(0.4$ to 0.8$)$ \\
\hline Classic or probable Katz hand diagram & 1 & $64 \%$ & $73 \%$ & 2.4 (1.6 to 3.5$)$ & $0.5(0.3$ to 0.7$) \dagger$ \\
\hline Hypalgesia & 2 & $15 \%$ to $51 \%$ & $85 \%$ to $93 \%$ & 3.1 (2.0 to 2.5$)$ & $0.7(0.5$ to 1.1$)$ \\
\hline Weak thumb abduction & 2 & $63 \%$ to $66 \%$ & $62 \%$ to $66 \%$ & $1.8(1.4$ to 2.3$)$ & $0.5(0.4$ to 0.7$)$ \\
\hline Phalen sign & 8 & $10 \%$ to $91 \%$ & $33 \%$ to $86 \%$ & $1.3(1.1$ to 1.6$)$ & $0.7(0.6$ to 0.9$)$ \\
\hline Nocturnal paresthesia & 3 & $51 \%$ to $84 \%$ & $27 \%$ to $68 \%$ & $1.2(1.0$ to 1.4$)$ & $0.7(0.5$ to 0.9$)$ \\
\hline
\end{tabular}

*Diagnostic terms defined in glossary.

†LRs are not combined. 